$20 \begin{aligned} & \text { Pesquisa Florestal Brasileira } \\ & \text { Brazilian Journal of Forestry Research } \\ & \text { https://www.embrapa.br/pfb }\end{aligned}$

\title{
Nota Científica \\ Ocorrência de Proscopiidae em Eucalyptus spp.: análise da infestação e distribuição espacial
}

\author{
Alexandre dos Santos ${ }^{1}$, Fernando Campos de Domenico², Otávio Peres Filho ${ }^{3}$, Rosane Segalla Soares ${ }^{3}$, Vagner Aniceto Teixeira ${ }^{1}$ \\ ${ }^{1}$ Instituto Federal de Educação, Ciência e Tecnologia de Mato Grosso, Campus Cáceres, Avenida dos Ramires, s/n, CEP 78.200-000, Mato Grosso, Brasil \\ ${ }^{2}$ Museu de Zoologia da Universidade de São Paulo, Avenida Nazaré, 481, Ipiranga, CEP 04.263-000, Sao Paulo, SP, Brasil \\ ${ }^{3}$ Universidade Federal do Mato Grosso, Departamento de Engenharia Florestal, Av. Fernando Corrêa da Costa, 2367, CEP 78.125-070, Cuiabá, MT, Brasil
}

\section{"Autor correspondente: \\ alexandre.santos@cas.ifmt.edu.br}

Termos para indexação:

Tetanorhynchus smithi

Proteção florestal

Taquarinha

Index terms:

Tetanorhynchus smithi

Forest protection

Stickbugs

\section{Histórico do artigo:}

Recebido em 07/05/2014

Aprovado em 12/12/2014

Publicado em 31/03/2015

doi: 10.4336/2015.pfb.35.81.706
Resumo - O objetivo desse estudo foi relatar a ocorrência de proscopídeos e quantificar a infestação e a distribuição espacial do ataque em um plantio experimental com três clones de eucalipto. No plantio foram utilizadas mudas de eucalipto dos clones dos híbridos GG100 de Eucalyptus urophylla x Eucalyptus grandis, H13 de Eucalyptus urophylla x Eucalyptus grandis e VM01 de Eucalyptus urophylla x Eucalyptus camaldulensis, dispostas em três blocos com 512 mudas, em espaçamento de $3 \mathrm{~m}$ x 2 m, no município de Cáceres, Mato Grosso, Brasil. Dois meses após o plantio, foi detectada a infestação na área por proscopídeos, os quais foram coletados para identificação. As plantas atacadas foram georeferenciadas e as injúrias quantificadas. A determinação do padrão espacial do ataque pelos proscopídeos foi realizada com os dados de posição das plantas danificadas, que foram submedidas à análise por quadrats utilizando-se os índices de dispersão binomial e de Morisita. Todos os indivíduos coletados foram identificados como Tetanorhynchus smithi Rehn, 1904 (Orthoptera: Proscopiidae), tendo a infestação diferida significativamente entre os três clones de eucalipto, com distribuição espacial do ataque nas mudas das plantas do tipo agregado. Esta foi a primeira ocorrência desta espécie de proscopídeo em eucalipto no estado de Mato Grosso.

\section{Occurrence of proscopiidae in Eucalyptus spp.: analysis of the infestations and spatial distribution}

\begin{abstract}
The objective of this research was to report the occurrence of proscopiidae and to quantify its infestation and spatial distribution in one experimental plantation of eucalypt clones. The study was conducted in an experimental field with eucalypt hybrids of Eucalyptus urophylla x Eucalyptus grandis (clones GG100), Eucalyptus urophylla $\mathrm{x}$ Eucalyptus grandis (H13) and of Eucalyptus urophylla $\mathrm{x}$ Eucalyptus camaldulensis (VM01). The plantation was established in three continuous blocks with 512 plants with $3 \mathrm{~m} \times 2$ $\mathrm{m}$, in Cáceres municipality, Mato Grosso State, Brazil. Infestation of proscopiidae insects was detected two months after planting. The insects were collected for identification. The attacked trees were geo-referenced and the injuries were quantified. The spatial pattern of proscopiidae he attack was determinated using the location information of the damaged plants, which have been submitted to a quadrats analyses using Morisita and binomial dispersion indexes. All the collected insects have been identified as Tetanorhynchus smithi Rehn, 1904 (Orthoptera, Proscopiidae). The infestation differed significantly among the three eucalypts clones and aggregated spatial pattern of attack was observed. This is the first report of this species of proscopiidae in eucalyptus in Mato Grosso State.
\end{abstract}


O eucalipto é a espécie florestal exótica mais amplamente cultivada no Brasil (Anuário..., 2012) e, provavelmente devido a sua proximidade taxonômica com diversas espécies vegetais brasileiras, essa planta tem sido frequentemente atacada por diversos grupos de insetos nativos da fauna neotropical (Holtz et al., 2003; Santos et al., 2003). Os extensos plantios homogêneos e contínuos de eucalipto, distribuídos por todo o território brasileiro, vêm exercendo forte pressão de seleção sobre esses insetos. A alta disponibilidade de alimento, aliada à baixa diversidade vegetal, tem provocado uma interferência no equilíbrio ecológico, causando alterações na diversidade e na estrutura da comunidade de toda a entomofauna (Andow, 1991). Essas mudanças têm levado a uma diminuição ou até mesmo à extinção local de algumas espécies (Russell, 1989; Tscharntke, 1992), ao mesmo tempo em que têm provocado um aumento populacional descontrolado de outras espécies, que atingem a condição de pragas (Hunter, 2002; Dalin et al., 2009).

Os plantios de eucalipto em sua fase inicial de desenvolvimento estão especialmente sujeitos a injúrias causadas por formigas cortadeiras, lagartas desfolhadoras e cupins subterrâneos (Zanuncio et al., 1998; Zanetti et al., 2000; Santos et al., 2011). Algo mais raro, no entanto, é o relato de ataques a esses plantios por insetos da família Proscopiidae, ortópteros popularmente conhecidos como taquarinhas ou manés-magros, cujos representantes são originários e exclusivos da América do Sul (Ferreira, 1978). Apenas duas espécies de proscopídeos são relatadas na literatura como pragas desse tipo de cultivo: Stiphra robusta Mello-Leitão, 1939, na região nordeste do país (Haji et al., 1985); e Pseudastroma multispinosa (Brunner von Wattenwyl, 1890) (sinônimo sênior de Tetanorhynchus leonardosi Mello-Leitão, 1939), na região central (Flechmann \& Ottati, 1997).

Esse trabalho tem como objetivo relatar uma nova ocorrência de ataque de proscopídeo em plantações de eucalipto, em um plantio experimental em Cáceres, MT, com três clones distintos de eucalipto, e analisar as injúrias e a distribuição desse inseto na região afetada.

O estudo foi realizado no Instituto Federal de Educação, Ciência e Tecnologia do Mato Grosso (IFMT) (5740'51'”; 16¹1'42"S e altitude de $117 \mathrm{~m}$ ), município de Cáceres, MT. A vegetação original é de Savana Florestada, clima tropical chuvoso com verão úmido e inverno seco tipo Awc pela classificação de Köppen. A média anual de temperatura é de $31,9^{\circ} \mathrm{C}$, o relevo é plano e o solo é classificado como Latossolo
Vermelho-Amarelo distrófico (Passos et al., 2006).

Em dezembro de 2012 foi implantado um povoamento experimental de eucalipto com mudas de clones dos híbridos GG100 de Eucalyptus urophylla x Eucalyptus grandis, $\mathrm{H} 13$ de Eucalyptus urophylla x Eucalyptus grandis e VM01 de Eucalyptus urophylla x Eucalyptus camaldulensis. As plantas foram dispostas em três blocos contínuos com 512 mudas de cada material genético, arranjadas em um espaçamento de $3 \mathrm{~m}$ x $2 \mathrm{~m}$, totalizando uma área de 0,92 ha. Dois meses após o plantio, foi detectada a infestação na área pelos proscopídeos, os quais foram coletados para identificação. Além disso, foi registrada a presença e ausência dos insetos e injúrias em cada uma das plantas, sendo os dados de posição das mudas posteriormente georeferenciados.

Para a determinação do padrão espacial do ataque em diferentes escalas espaciais, os dados georeferenciados das mudas com injúrias causadas pelo proscopídeos foram submedidas à análise por quadrats utilizando-se os índices de dispersão binomial (Db) e de Morisita (Id). Cada quadrat foi definido como uma área retangular dentro do plantio, que abrange um determinado número de plantas na linha e entre linhas. $O$ índice de dispersão binomial é dado por $\mathrm{D}_{\mathrm{b}}=\mathrm{V}_{\mathrm{obs}} / \mathrm{V}_{\mathrm{bt}}$, sendo $\mathrm{V}_{\mathrm{obs}}$ a razão da variância observada $\mathrm{e}_{\mathrm{bt}}$ a variância teórica (Krainski \& Ribeiro Junior, 2006). O índice de Morisita é dado por $\mathrm{I}_{\mathrm{d}}=\mathrm{N}\left[\sum x^{2}-\sum x / \sum(x)^{2}-\sum x\right]$, sendo $\mathrm{N}$ o número de quadrats e $x$ o número de plantas atacadas nos quadrats (Morisita, 1962). Para ambas as análises foram considerados quadrats de $2 \mathrm{~m} \times 2 \mathrm{~m}, 3 \mathrm{~m} \times 3 \mathrm{~m}, 4 \mathrm{~m} \times 4 \mathrm{~m}, 5 \mathrm{~m} \times 5 \mathrm{~m}$, $6 \mathrm{~m} \times 6 \mathrm{~m}, 7 \mathrm{~m} \times 7 \mathrm{~m}$ e $8 \mathrm{~m} \times 8 \mathrm{~m}$. As análises foram realizadas com o emprego dos pacotes Rcitrus (Krainski \& Ribeiro Junior, 2006) e spastat (Baddeley \& Turner, 2005) por meio do software R (R Development Core Team, 2008).

Para comparar a intensidade do ataque entre os três diferentes clones de eucalipto, foi feita uma análise de variância e regressão utilizando-se modelos lineares generalizados com uma distribuição de erros binomial para a variável-resposta (Buckley et al., 2003; Crawley, 2005). Posteriormente, realizou-se a junção dos termos qualitativos não significativos, através de análises de contraste de modelos, para verificar a semelhança entre os diferentes clones de eucalipto, a partir do modelo completo. As análises foram realizadas por meio do software R (R Development Core Team, 2008).

Todos os indivíduos coletados foram identificados como Tetanorhynchus smithi Rehn, 1904 (Orthoptera, 
Proscopiidae). Essa espécie ocorre na região central do Brasil, na Bolívia e no Paraguai. Sua identificação foi feita com base na análise das características morfológicas externas e genitais. Trata-se de uma espécie muito semelhante à Tetanorhynchus humilis Giglio-Tos, 1987, diferindo dessa principalmente devido à constituição mais robusta do corpo e à condição inflada do meso e metatórax (Jago, 1989) (Figura 1).

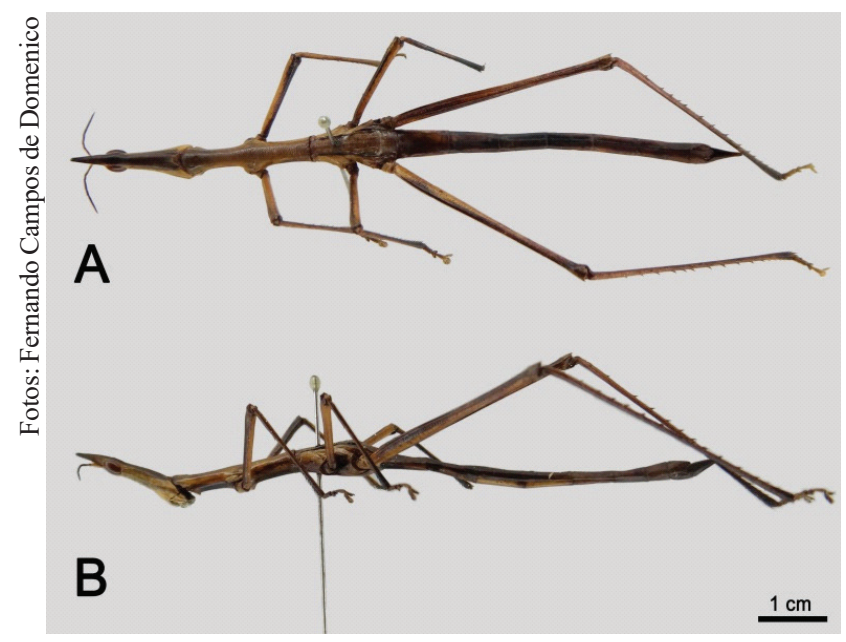

Figura 1. Taquarinha Tetanorhynchus smithi Rehn, 1904 (Orthoptera, Proscopiidae). A = vista dorsal e B = vista lateral. Cáceres, MT, 2012.

As injúrias provocadas às mudas de eucalipto apresentaram sucessão crescente, iniciando-se com o consumo de pequenas porções das bordas das folhas que evoluíram para o consumo total do limbo foliar, restando em alguns casos, apenas à nervura central (Figura 2).

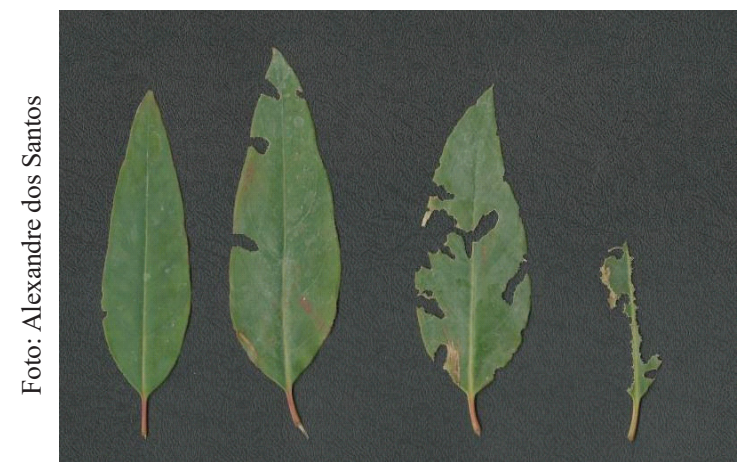

Figura 2. Sucessão das injúrias provocadas por Tetanorhynchus smithi em mudas de diferentes clones de Eucalyptus spp. Cáceres, MT, 2012.
A análise de variância evidenciou que a infestação por $T$. smithi diferiu significativamente entre os clones de eucalipto $(\chi 2=70,86 ; \mathrm{p}<0,0001)$. O clone H13 foi o mais atacado ( $68 \%$ das plantas), seguido por VM $01(56 \%)$ e o menos preferido foi o GG100 (42,19\%) (Figura 3). Flechtmann \& Ottani (1997) também relaram danos causados em Eucalyptus urophylla pela espécie de taquarinha Pseudastroma multispinosa em um plantio de três meses, mas em menor intensidade de ataque (5,8\%). Esses autores observaram danos também ao meristema das mudas, o que não foi observado no ataque aqui relatado. No entanto, mais estudos são necessários para se confirmar a causa desta diferença na preferência entre os diferentes materiais genéticos pelo inseto.

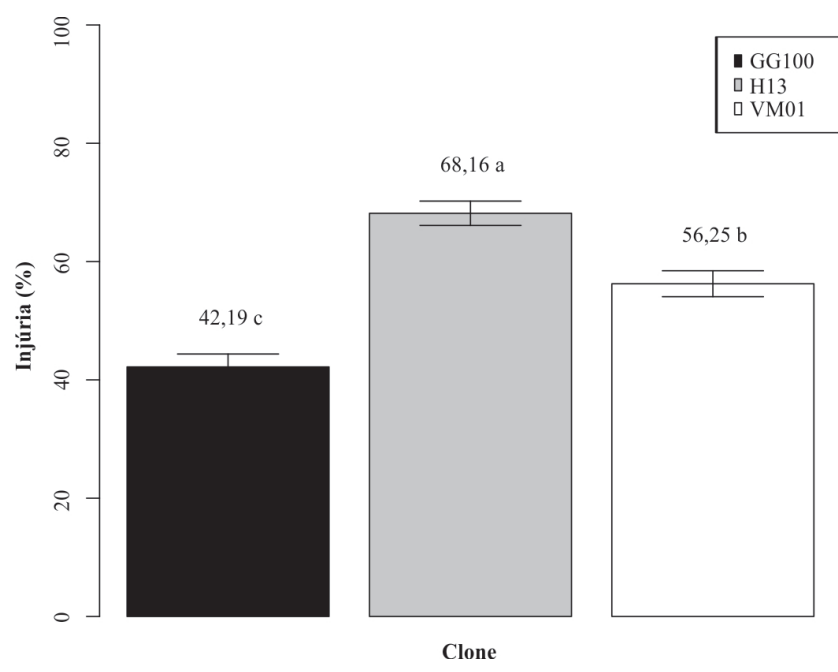

Figura 3. Injúria (\%) provocada por Tetanorhynchus smithi em mudas de clones dos híbridos GG100 (Eucalyptus urophylla $\mathrm{x}$ Eucalyptus grandis), H13 (Eucalyptus urophylla $\mathrm{x}$ Eucalyptus grandis) e VM01 (Eucalyptus urophylla x Eucalyptus camaldulensis). Cáceres, MT, 2012.

A distribuição espacial determinada com os resultados conjuntos dos três clones analisados demonstrou um padrão de distribuição agregado de ataque por T. smithi, com valores acima da unidade para o índice de dispersão binomial e de Morisita, considerando todos os tamanhos de quadrats adotados (Figura 4). O padrão de distribuição de um inseto sob o status de praga pode variar ao longo do tempo, sendo que no início da colonização na cultura a tendência da distribuição é aleatória, evoluindo para uma distribuição agregada e raramente atingindo a distribuição regular, quando os insetos atingem altas populações (Young \& Young, 1998). Para T. smithi, a variação na escala espacial utilizada na análise não 
levou a modificações no padrão da distribuição espacial, como observado para outras espécies (Barbosa, 1992), demonstrando uma colonização agregada e não aleatória do recurso alimentar, por essa espécie de proscopídeo.

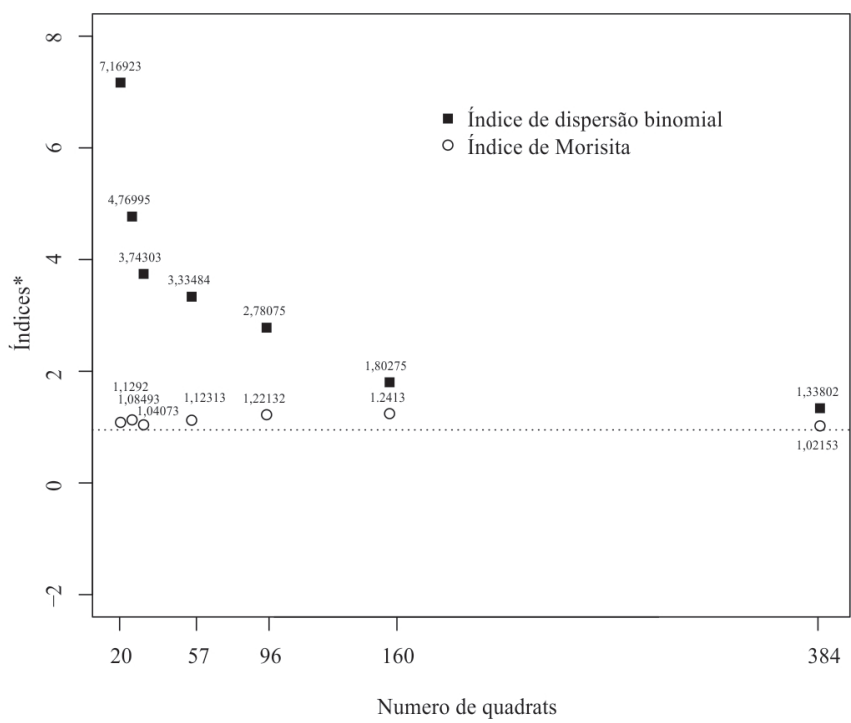

Figura 4. Índice de dispersão binomial e índice de Morisita para a distribuição espacial de Tetanorhynchus smithi em mudas de eucalipto.*(p<0,05). Cáceres, MT, 2012.

\section{Conclusões}

As plantações de eucalipto estão sujeitas a pragas até então desconhecidas para a Região Centro-Oeste, como os proscopídeos da espécie Tetanorhynchus smithi. Alguns clones dessa planta são mais preferidos que outros, no entanto a razão disso permanece desconhecida. A distribuição espacial do ataque de $T$. smithi em mudas de eucalipto mostrou padrão agregado.

\section{Referências}

ANUÁRIO Estatístico da ABRAF: ano base 2012. Disponível em: $<$ http://www.abraflor.org.br/estatisticas/ABRAF12/ABRAF12-BR. pdf $>$. Acesso: 20 de outubro de 2012.

ANDOW, D. A. Vegetational diversity and arthropod population response. Annual Review of Entomology, v. 36, p. 561-586, 1991.

BADDELEY, A. J.; TURNER, R. Spatstat: an R package for analyzing spatial point patterns. Journal of Statistical Software, v. 12, p. 1-42, 2005.

BARBOSA, J. C. A amostragem sequencial. In: FERNANDES, O. A.; CORREIA, A. C. B.; BORTOLI, S. A. (Ed.). Manejo integrado de pragas e nematoides. Jaboticabal: FUNEP, 1992. p. 205-211.
BUCKLEY, Y. M.; BRIESE, D. T.; REES, M. Demography and management of the invasive plant species Hypericumperforatum, I, using multi-level mixed-effects models for characterizing growth, survival and fecundity in a long- term data set. Journal of Applied Ecology, Oxford, v. 40, n. 3, p. 481-493, 2003.

CRAWLEY, M. J. Statistics an introduction using R. Jonh Wiley and Sons, Chinchester, 2005. 327 p.

DALIN, P.; KINDVALL, O.; BJÖRKMAN, C. Reduced population control of an insect pest in managed willow monocultures, PLoS ONE, v. 4, n. 5, p. e5487, 2009.

FERREIRA, A. Contribuição ao estudo da evolução dos Proscopiidae (Orthoptera:Proscopoidea). Studia Entomologica, v. 20, p. 1-4, 1978.

FLECHTMANN, C. A. H.; OTTATI, A. L. T. Tetanorhynchus leonardosi (Mello-Leitão) (Orthoptera: Proscopiidae), nova praga em eucaliptos. Anais da Sociedade Entomológica do Brasil, Londrina, v. 26, n. 3, p. 583-587, 1997.

HAJI, F. N. P.; SOUZA, S. M. de; TOSCANO, J. C. Ação de diversos inseticidas sobre Stiphra robusta Mello-Leitão, 1939. (Orthoptera, Proscopiidae) em Eucalyptus citriodora. Anais da Sociedade de Entomologia do Brasil, Jaboticabal, v. 14, n.1, p. 23-28, 1985.

HOLTZ, A. M.; OLIVEIRA, H.G.; PALLINI, A.; MARINHO, J. S.; ZANUNCIO, J. C.; OLIVEIRA, C. L. Adaptação de Thyrinteina arnobia em novo hospedeiro e defesa induzida por herbívoros em eucalipto. Pesquisa Agropecuária Brasileira, v. 38, n. 4, p. 453458, 2003.

HUNTER, M. D. Ecological causes of pest outbreaks. In: PIMENTEL, D. (Ed.). Encyclopedia of Pest Management. Boca Raton: CRC Press, 2002. p. 214-217.

JAGO, N. D. The genera of the Central and South American grasshopper family Proscopiidae (Orthoptera: Acridomorpha). EOS: revista española de entomología, Madri, v. 65, p. 249-307, 1989.

KRAINSKI, E. T.; RIBEIRO JUNIOR, P. J. Rcitrus: R functions for analysis of citrus disease data. $R$ package version 0.3-1, 2006. Disponível em: <http://www.leg.ufpr.br/Rcitrus $>$.

MORISITA, M. Id-index, a measure of dispersion of individuals. Researches on Population Ecology, v. 4, n. 1, p. 1-7, 1962.

PASSOS, C. A. M.; BUFULIN JÚNIOR, L.; GONÇALVES, M. R. Avaliação silvicultural de Tectona grandis L.f., em Cáceres - MT, Brasil: resultados preliminares. Ciência Florestal, v. 16, n. 2, p. 225-232, 2006.

R DEVELOPMENT CORE TEAM R. A language and environment for statistical computing. Vienna: R Foundation for Statistical Computing, 2008.

RUSSELL, E. P. Enemies hypothesis: a review of the effect of vegetational diversity on predatory insects and parasitoids. Environmental Entomology, v. 18, n. 4, p. 590-599, 1989.

SANTOS, A.; ZANETTI, R.; FERNANDES, B.V.; SERRÃO, E. J.; ZANUNCIO, J. C. Subterranean termites (Insecta: Isoptera) sampled in sandy and sandy-clay soils at Minas Gerais Cerrado, Brazil. Sociobiology, v. 57, p. 633-643, 2011. 
SANTOS, G. P.; ZANUNCIO, T. V.; FREITAS, M .F.; ALVES, J. P.; ZANUNCIO, J. C. Biologia comparada e consumo foliar de Anisodes sp. (Lepidoptera: Geometridae) em folhas novas e velhas de Eucalyptus grandis W. Hill ex Maid (Myrtaceae) e Psidium guajava L. (Myrtaceae). Bioscience Journal, v. 19, n. 3, p. 69-76, 2003.

TSCHARNTKE, T. Fragmentation of Phragmites habitats, minimum viable population size, habitat suitability, and local extinction of moths, midges, flies, aphids, and birds. Conservation Biology, v. 6, n. 4, p. 530-536, 1992.

YOUNG, L. J.; YOUNG, J. H. Statistical Ecology: a population perspective. Boston: Kluwer Academic Publishers, 1998. 565 p.
ZANETTI, R; VILELA, E. F.; ZANUNCIO, J. C.; LEITE, H. G.; FREITAS, G. D. Influência da espécie cultivada e da vegetação nativa circundante na densidade de sauveiros em eucaliptais. Pesquisa Agropecuária Brasileira, Brasília, v. 35, n. 10, p. 1911-1918, 2000.

ZANUNCIO, J. C.; MEZZOMO, J. A.; GUEDES, R.N.C.; OLIVEIRA, A. C. Influence of strips of native vegetation on Lepdotera associated with Eucalyptus cloeziana in Brazil. Forest Ecology and Mangement, Amsterdam, v. 108, n. 1/2, p. 85-90, 1998. 
